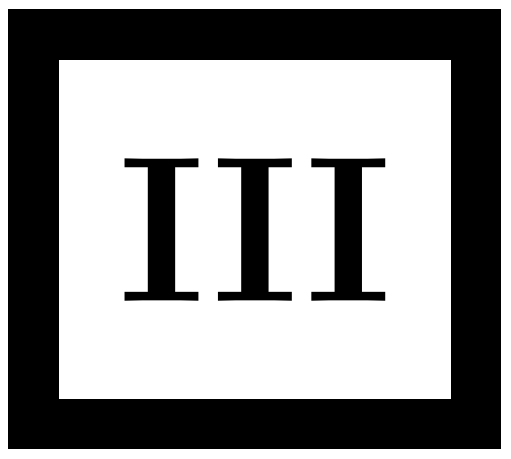

Publication III

S. Suihkonen, O. Svensk, T. Lang, H. Lipsanen, M. A. Odnoblyudov and V. E. Bougrov, The effect of In GaN/GaN MQW hydrogen treatment and threading dislocation optimization on GaN LED efficiency, Journal of Crystal Growth 298, 740 (2007).

Reprinted with permission from the publisher (c) 2007 Elsevier 


\title{
The effect of InGaN/GaN MQW hydrogen treatment and threading dislocation optimization on GaN LED efficiency
}

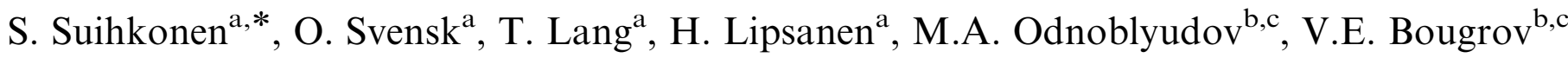 \\ ${ }^{a}$ Optoelectronics Laboratory, Helsinki University of Technology, FIN-02150 TKK, Finland \\ ${ }^{\mathrm{b}}$ A. F. Ioffe Physico-Technical Institute, Russian Academy of Science, 194021 St. Petersburg, Russia \\ ${ }^{\mathrm{c}}$ OptoGaN Oy, Tietotie 3, 02150 Espoo, Finland
}

Available online 28 November 2006

\begin{abstract}
We report on the effect of GaN buffer threading dislocation (TD) optimization and InGaN/GaN quantum well (QW) hydrogen ( $\mathrm{H}_{2}$ ) treatment on the efficiency of GaN light emitting diodes (LEDs) operating in the spectral range from 400 to $500 \mathrm{~nm}$. A tenfold reduction of the TD density in the GaN buffer increased the efficiency of blue LEDs operating at high current density, while in green LEDs it had very little effect. The reduced TD density also increased the compressive strain in the InGaN QWs, and caused blue shift to the electroluminescence (EL) peak wavelength. The $\mathrm{H}_{2}$ treatment of the QWs increased strain inside the MQW stack. It was possible to apply the $\mathrm{H}_{2}$ treatment only to UV LEDs, as the increased strain in blue and green LEDs caused relaxation of the MQW stack. Although this resulted in smooth surface morphology of the MQW stack, it did not lead to any increase in the efficiency of the UV LEDs.

(C) 2006 Published by Elsevier B.V.
\end{abstract}

PACS: 73.21.Fg; 78.55.Cr; 78.60.Fi; 85.60.Jb

Keywords: A3. Metalorganic chemical vapor deposition; B1. InGaN; B1. Quantum wells; B3. Light-emitting diodes

\section{Introduction}

Major developments in III-nitride semiconductors have led to the commercial production of InGaN-based multiquantum-well (MQW) light-emitting diodes (LEDs) and laser diodes [1]. GaN MQW LEDs use InGaN as the material for quantum well (QW) layers and $\mathrm{GaN}$ as the material for barrier layers, sapphire and silicon carbide are commonly used as substrates.

A large number of threading dislocations (TD) originate from the large lattice constant difference of $\mathrm{GaN}$ and sapphire. These TDs propagate through the entire LED structure, including the MQW stack. Recent experimental observations by Hangleiter et al. [2] give strong arguments that the high radiative efficiency of $\mathrm{InGaN} / \mathrm{GaN}$ QWs is due to a potential barrier that is formed around the TDs. This causes screening of the dislocations and prevents carriers from being trapped by them. In this case, the

\footnotetext{
${ }^{*}$ Corresponding author. Tel.: + 35894512325 ; fax: +35894513128 .

E-mail address: sami.suihkonen@hut.fi (S. Suihkonen).
}

recombination mechanism should be determined by a competition between radiative recombination in the InGaN wells and tunnelling through the potential barriers that screen the dislocations. As both of these depend on the In content, the effect of TD density on the LED efficiency should be different in the case of high and low In content in the QWs.

In addition the MOVPE growth regimes for high quality $\mathrm{GaN}$ and for InGaN with high indium content are essentially different. This results in compromises in the growth process of the LED active region. When grown in these conditions, the surface of a thin GaN barrier located on top of an InGaN QW layer is characterized by the presence of pits with In-rich inclusions located in the center of the pits [3]. The presence of these few nanometer high inclusions deteriorates the morphology of the $\mathrm{InGaN} / \mathrm{GaN}$ MQW structure and can lead to premature thermal degradation of the InGaN wells [4]. This imposes limitations on the thermal budget of $\mathrm{p}-\mathrm{GaN}$ layer growth and post growth thermal annealing of the LED structure. Therefore, various growth techniques have been employed 
that strive for smooth planar morphology and sharp interfaces within the InGaN/GaN stack. The growth of GaN barriers at elevated temperature [5], and introducing hydrogen $\left(\mathrm{H}_{2}\right)$ during barrier growth [6,7] are believed to be the most efficient approaches for improving the surface morphology and thermal stability of InGaN/GaN QWs.

In this work, we have performed an extensive study on the role of TD density and MQW $\mathrm{H}_{2}$ treatment on the efficiency of GaN LEDs operating in spectral range from 400 to $500 \mathrm{~nm}$. It is shown that the increased strain incorporated in the low TD density GaN buffer causes a blue shift in the electroluminescence (EL) peak wavelength of the LEDs. The reduction of TD density was found to increase the efficiency of blue LEDs operating at high current density, while in green LEDs it had very little effect. The $\mathrm{H}_{2}$ treatment of the QWs was found to increase strain in the MQW stack. It was possible to apply the $\mathrm{H}_{2}$ treatment only to UV LEDs, as the increased strain in blue and green LEDs caused relaxation of the MQW stack. Although the $\mathrm{H}_{2}$ treatment improves the surface morphology of the MQW stack, we did not observe any significant increase in the efficiency of the UV LEDs.

\section{Experimental procedure}

The LED structures were grown on sapphire substrates in a $3 \times 2$ in Thomas Swan Close Coupled Showerhead MOVPE reactor. Trimethylgallium (TMGa), ammonia $\left(\mathrm{NH}_{3}\right)$, trimethylindium (TMI), and trimethylaluminum (TMA) were used as gallium, nitrogen, indium, and aluminum sources, respectively. Silane $\left(\mathrm{SiH}_{4}\right)$ and bis(cyclopentadienyl)magnesium $\left(\mathrm{Cp}_{2} \mathrm{Mg}\right)$ were used for $\mathrm{n}$-, and p-type doping, respectively. Two different processes for GaN buffer growth were used. The standard "two-step" process resulted in TD density in the excess of $6 \times 10^{8} \mathrm{~cm}^{-2}$. Low dislocation density buffer was grown with the application of a multistep nucleation layer technique [8], which enables TD density of $7 \times 10^{7} \mathrm{~cm}^{-2}$ to be achieved.

The MQW stack of the LED structure consisted of 10 pairs of $3 \mathrm{~nm}$ thick InGaN QWs and $25 \mathrm{~nm}$ thick GaN barriers. Growth temperature of the InGaN wells and $\mathrm{GaN}$ barriers was varied between 800 and $875^{\circ} \mathrm{C}$ to achieve In content between $18 \%$ and $5 \%$. This corresponded to EL peak wavelength from 500 to $400 \mathrm{~nm}$. $\mathrm{N}_{2}$ was used as a carrier gas during the growth of MQW stack. Hydrogen treatment was performed by introducing $\mathrm{H}_{2}$ during the growth of $\mathrm{GaN}$ barriers. The flow ratio of $\mathrm{H}_{2}$ and $\mathrm{N}_{2}$ during the treatment was 0.02 . In the $\mathrm{H}_{2}$ treated samples the $\mathrm{GaN}$ barriers were grown at $920^{\circ} \mathrm{C}$. To compensate the In desorption caused by $\mathrm{H}_{2}$, the growth temperature of the wells was decreased to achieve the correct wavelength. The MQW stack was capped by a $20 \mathrm{~nm}$ thick AlGaN layer with $12 \% \mathrm{Al}$ content. During the growth of the capping layer the temperature was increased to the p-GaN growth temperature of $1050{ }^{\circ} \mathrm{C}$ and the carrier gas was switched to $\mathrm{H}_{2}$.
The optical properties of the LEDs were characterized by EL measurements. For the EL measurements $1 \mathrm{~mm}^{2}$ metallic In contacts were soldered to $\mathrm{p}$ - and $\mathrm{n}$ - layers on the LED wafers. EL spectra and intensity were measured through the sapphire substrate by a backside detector. The In content of the QWs was determined by X-ray diffraction (XRD) measurements. The TD densities of different types of $\mathrm{GaN}$ buffers were confirmed by atomic force microscopy measurements of etched surfaces.

\section{Results and discussion}

The effect of GaN buffer TD density on the GaN LED efficiency was studied by growing a similar set of UV, blue and green LED structures on a regular "two-step" GaN buffer with TD density of $6 \times 10^{8} \mathrm{~cm}^{-2}$ and on a low dislocation density buffer with TD density of $7 \times 10^{7} \mathrm{~cm}^{-2}$. Fig. 1 shows the EL spectra measured from UV (400 nm), blue $(460 \mathrm{~nm})$ and green $(500 \mathrm{~nm})$ LEDs grown on both types of buffers operating at $20 \mathrm{~mA}$ current. It can be seen that the emission from the LED structures grown on low TD density buffer is blue shifted $10 \mathrm{~nm}$, and the emission peak full width half maximum (FWHM) is decreased by approximately $20 \%$. The intensity of the blue LED structure grown on low TD density buffer is increased by $30 \%$, but the intensity green and UV structures is decreased by $40 \%$ and $60 \%$, respectively. XRD scans showed no difference in the In content or QW quality between the structures grown on "two-step" and low TD density buffers. The blue shift of the LEDs grown on multistep buffers can be explained by the increased compressive strain in the buffer layer that adds to the strain inside the MQW stack. Compressive strain has been found to cause blue shift of the PL emission of InGaN QWs, and also to decrease PL intensity [9]. As a similar structure was used for green, blue and UV LEDs, the poor

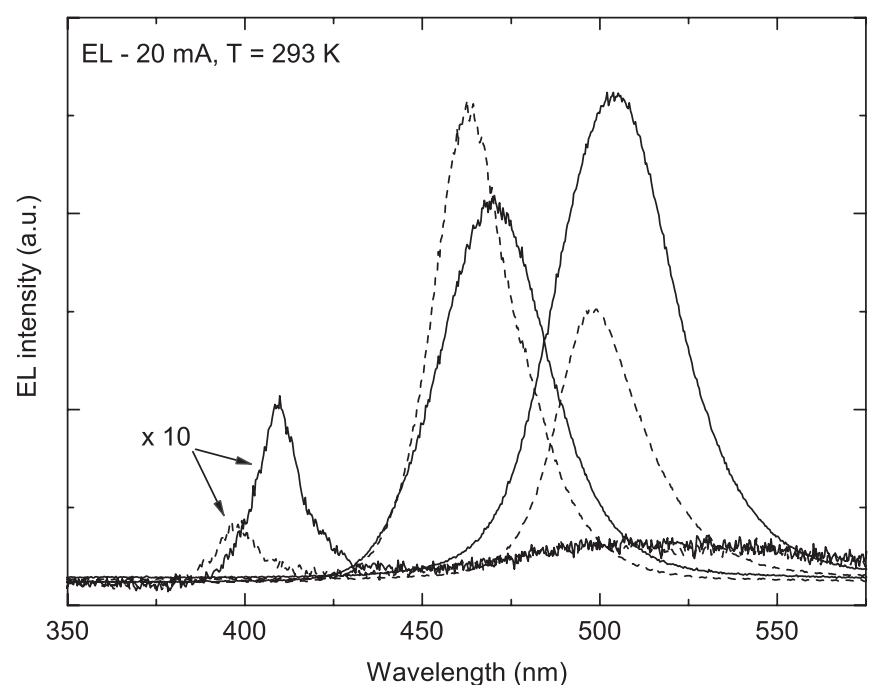

Fig. 1. Electroluminescence spectra of LEDs grown on "two-step" buffer (straight line) and on low TD density buffer (dashed line). 
performance of UV LEDs is most likely caused by nonoptimal MQW and capping structure for short wavelengths.

The EL intensities of blue and green LED structures grown on both types of buffers were measured as a function of operating current (see Fig. 2). Saturation of the EL intensity at high current is observed in the blue LED. The tenfold reduction of TD density increased the saturation threshold from 100 to $150 \mathrm{~mA}$, this corresponds to current densities of 10 and $15 \mathrm{~A} / \mathrm{cm}^{2}$, respectively. Also the maximum EL intensity was increased by $70 \%$. No saturation was observed in the green LED structure grown on "two-step" or on low TD buffer. This supports the model of recombination mechanism being determined by a competition between the radiative recombination in the InGaN wells and tunnelling through the potential barriers that screen the dislocations. The probability of carriers tunnelling to the dislocations increases with increasing carrier density but decreases with increasing potential barrier height caused by the higher In content. Therefore, the reduction of dislocation density has the biggest effect in LED efficiency at high current densities when the In content of the QWs is small. Unfortunately, due to the poor performance of our UV LED structure we were unable to verify these results with small In content QWs.

To smoothen the rough surface morphology of the MQW stack, in situ $\mathrm{H}_{2}$ treatment together with the growth of barriers at elevated temperatures was done on a one set of LED samples. This process has been proven improve the surface morphology of the MQW stack, without deteriorating the optical quality of the QWs [10]. With green and blue LEDs this treatment resulted in relaxation of the QWs during the growth of the MQW stack or $\mathrm{p}-\mathrm{GaN}$ layer over the stack. This was observed by a decrease of oscillation amplitude and reflection signal of the in situ reflectometer of the MOVPE system. The relaxation most likely resulted

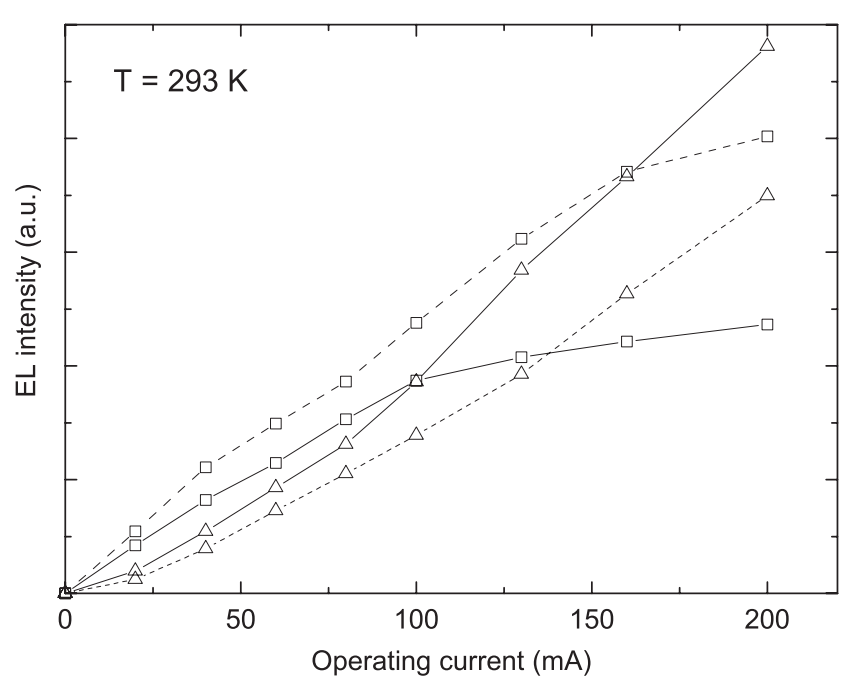

Fig. 2. EL intensity of blue (squares) and green (triangles) LEDs grown on "two-step" (solid line) and on low TD density buffer (dashed line) as a function of operating current.

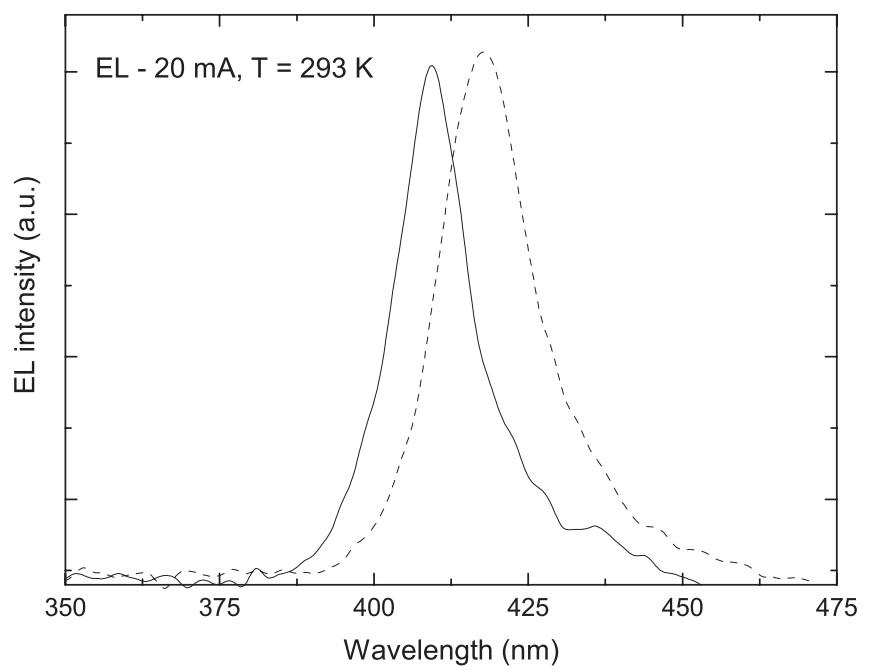

Fig. 3. EL spectra of UV LED with $\mathrm{H}_{2}$ treatment (dashed line) and without $\mathrm{H}_{2}$ treatment (solid line).

due to the build up of strain in the MQW stack. We believe this is caused by the removal of In rich bumps that otherwise release strain inside the QWs. However, the $\mathrm{H}_{2}$ treatment could be used for UV LED structures where In content is low. Surprisingly in UV LEDs the $\mathrm{H}_{2}$ treatment caused a $20 \%$ increase of EL peak FWHM and no improvement of EL intensity (Fig. 3). This supports our previous findings that $\mathrm{In}$ rich clusters in the $\mathrm{InGaN} / \mathrm{GaN}$ MQW structure do not play a significant role in PL or EL emission of the QWs [10].

\section{Conclusions}

We have studied the effect of TD density and MQW $\mathrm{H}_{2}$ treatment on GaN LED efficiency. The effect of the TD density varied with the In content of the QWs and the current density of the device. Saturation of EL intensity at high current was observed in blue LEDs, but not in green LEDs. In blue LEDs, the decrease of the TD density by an order of a magnitude increased the saturation current threshold current by $50 \%$. As the height of the potential barriers formed around the TDs is determined by the In content of the QWs, and the probability of carriers tunnelling through these barriers depends on the current density of the device, the reduction of TD density has the biggest effect on LED efficiency at high current densities and when the In content of the QWs is small. The reduction of TD density of the GaN buffer was also found to increase the compressive strain in the InGaN QWs, and cause blue shift to the EL peak wavelength in all the studied LED structures.

Although $\mathrm{H}_{2}$ treatment could be used to improve the surface morphology of the MQW stack, it could not be used to improve the efficiency of GaN LEDs. The $\mathrm{H}_{2}$ treatment removes In rich bumps from the MQW structure, that in our belief causes additional build up of strain inside the MQW stack. This increased strain caused 
by the relaxation of blue and green LED MQW structures during the growth. It was possible to apply the $\mathrm{H}_{2}$ treatment only to UV LEDs, where it did not lead to any increase in the efficiency of the devices.

\section{References}

[1] S. Nakamura, G. Fasol, The Blue Laser Diode, Springer, Berlin 1997.

[2] A. Hangleiter, et al., Phys. Rev. Lett. 95 (2005) 127402.

[3] I.-H. Kim, H.-S. Park, Y.-J. Park, T. Kim, Appl. Phys. Lett. 73 (1998) 1634.
[4] H. Ishikawa, N. Nakada, M. Mori, G.Y. Zhao, T. Egawa, T. Jimbo, M. Umeno, Jpn. J. Appl. Phys. Part 240 (2001) L1170.

[5] F. Scholz, J. Off, E. Fehrenbacher, O. Gfrörer, G. Brockt, Phys. Status Solidi A 180 (2000) 315.

[6] S.M. Ting, et al., J. Appl. Phys. 94 (2003) 1461.

[7] Y.-T. Moon, et al., J. Appl. Phys. 89 (2001) 6514.

[8] T. Lang, et al., J. Crystal Growth 277 (2005) 64.

[9] M.E. Aumer, S.F. LeBoeuf, S.M. Bedair, M. Smith, J.Y. Lin, H.X. Jiang, Appl. Phys. Lett. 77 (2000) 821.

[10] S. Suihkonen, T. Lang, O. Svensk, J. Sormunen, M. Sopanen, H. Lipsanen, M.A. Odnoblyudov, V.E. Bougrov, J. Crystal Growth, submitted for publication. 